\title{
PRODUCCIÓN DE ABONOS ORGÁNICOS CON LA UTILIZACIÓN DE ELODEA (Egeria densa) PRESENTE EN LA LAGUNA DE FúQUENE
}

\section{PRODUCTION OF ORGANIC FERTILIZERS WITH ELODEA (Egeria densa) PRESENT ON THE FUQUENE LAGOON}

\author{
Iveth Caro Lara ${ }^{1}$ \\ Zulma Romero Otálora ${ }^{1}$ \\ Rodrigo Lora Silva²
}

\section{RESUMEN}

La elodea (Egeria densa), un problema para lagos y lagunas en Colombia, puede ser materia prima para la producción de abonos orgánicos de bajo costo y de buena calidad. Para emplearla en la obtención de abonos orgánicos, se elaboraron fertilizantes a partir de seis formulaciones, así: 1) Elodea 50\%; cal dolomítica 20\%; levadura 5\%; melaza 5\%; gallinaza 20\%. 2) Elodea 60\%; cal dolomítica $10 \%$; levadura 3\%; melaza $8 \%$; gallinaza 19\%. 3) Elodea 70\%; cal dolomítica $15 \%$; levadura $2 \%$; melaza 3\%; gallinaza $10 \%$. 4) Formulación $1+$ lombricompuesto; 5) Formulación 2 + lombricompuesto y 6) Formulación 3 + lombricompuesto. Para cada uno de los abonos, se determinó el contenido total de $\mathrm{N}$, $\mathrm{P}_{2} \mathrm{O}_{5}, \mathrm{~K}_{2} \mathrm{O}, \mathrm{MgO}, \mathrm{CaO}$, carbón oxidable total, coliformes y relación C:N. Bajo condiciones de invernadero, se evaluó el efecto de los abonos en un Andisol sobre el cultivo de lechuga e igualmente, se monitoreó la temperatura y el $\mathrm{pH}$ a través del periodo de producción de los abonos. En general, el contenido de los elementos analizados

${ }^{1}$ Químico Industrial. Universidad de Ciencias Aplicadas y Ambientales - Corporación Tecnológica de Bogotá, Cra.21 No.53D - 35. Bogotá, D.C.

2 Ingeniero Químico, M.Sc. Docente-Investigador. Universidad de Ciencias Aplicadas y Ambientales U.D.C.A rodrigolorasilva@yahoo.com Dirección para correspondencia: Calle 222 No. 54-37, Bogotá, D.C. y la relación C: $N$ cumplen los requisitos para estos abonos. Hubo efecto significativo del contenido entre formulaciones y en el bioensayo para rendimiento entre formulaciones y dosis, siendo la formulación tres la de más alto rendimiento. De igual manera hubo efecto significativo de fuentes y de dosis en el contenido de $\mathrm{N}$ P-K en la lechuga. El pH de los seis abonos fue de 7,00 a 7,30, considerado apropiado. Se demostró la factibilidad de producción de abonos orgánicos de buena calidad a partir de elodea.

Palabras clave: Fertilización orgánica, compostación, lombricompuesto, elodea, bioensayo.

\section{SUMMARY}

Elodea (Egeria densa), a problem in lakes and lagoons of Colombia, could be a raw material to produce cheap and good quality organic fertilizers. To evaluate the elodea for organic compost production, fertilizers were elaborated with six formulations: 1) Elodea 50\%; dolomitic lime 20\%; yeast $5 \%$; melasse $5 \%$; hene manure $20 \%$. 2) Elodea $60 \%$; dolomitic lime $10 \%$; yeast $3 \%$; melasse $8 \%$; hene manure 19\%. 3) Elodea 70\%; dolomitic lime 15\%; yeast $2 \%$; melasse 3\%; hene manure 10\%. 4) Formulation 1 + earthworm fertilizer. 5) Formulation $2+$ earthworm fertilizer. Formulation y 6) Formulation $3+$ earthworm fertilizer. For each fertilizer the total content of $\mathrm{N}, \mathrm{P}_{2} \mathrm{O}_{5}$, $\mathrm{K}_{2} \mathrm{O}, \mathrm{MgO}, \mathrm{CaO}$, total oxidised carbon, coliforms and $\mathrm{C}$ : $N$ were analyzed. Under greenhouse conditions the effect of the fertilizers were evaluated in an Andisol and with 
lettuce as indicator. During production of the fertilizer temperature and $\mathrm{pH}$ were observed. The results showed significant effect of the elements among organic fertilizer. The composition of these fertilizers was according to the requisites for Colombian Organic Fertilizers. The greenhouse experiment showed significant effect of sources and levels on yield and N-P-K content of the lettuce. In general, the formulation three was the best. The $\mathrm{pH}$ of the fertilizers varied from 7.0 to 7.3 , considered adequate. The feasibility to produce good quality organic fertilizers from elodea was demonstrated.

Key words: Organic fertilizer, compost, earth worm manure, elodea, bioassay.

\section{INTRODUCCIÓN}

La laguna de Fúquene tiene gran importancia para las poblaciones, quienes dependen del recurso hídrico para su alimentación, riego de los cultivos, agua para consumo y, en general, para resolver parte de sus necesidades. Actualmente, la cuenca de la laguna presenta problemas socioeconómicos y ambientales, tales como pérdida de volumen del cuerpo de agua; proliferación de plantas acuáticas (eutrofización); arrastre del suelo por la lluvia, debido a prácticas agropecuarias y mineras inadecuadas, que genera sedimentación en la laguna; pesca y cacería indiscriminada y contaminación del espejo del agua causada, principalmente, por la invasión de plantas acuáticas que crean problemas al ecosistema de la laguna, como la reducción de la capacidad de embalse y el deterioro de la calidad del agua por falta de oxígeno, por la descomposición del material vegetal que, puede producir sustancias tóxicas como el ácido sulfhídrico (JICA, 1999).

La laguna, se encuentra ubicada en el departamento de Cundinamarca, sobre la vertiente occidental de la cordillera oriental, a los $50^{\circ} 28^{\prime} 12^{\prime \prime}$ de latitud Norte y $73^{\circ} 44^{\prime} 14^{\prime \prime}$ de latitud Oeste a $2543 \mathrm{msnm}$, en la jurisdicción de los municipios de Fúquene, Ráquira, Guachetá, San Miguel de Sema y Susa (JICA, 1999; Valderrama E Mosquera, 1979; CAR, 2000). El río Ubaté, luego de reunirse con tributarios como Susa, Cucunubá y Lenguazaque, alimentan la laguna. Posee una área aproximada de $30 \mathrm{~km}^{2}$ y una área de drenaje de $992 \mathrm{~km}^{2}$; la temperatura promedia es de $13^{\circ} \mathrm{C}$, precipitación $638 \mathrm{~mm}$, con una humedad relativa promedia mensual de $75 \%$. En cuanto al estado de conservación de la laguna es posible observar en el tiempo un aumento de la invasión de plantas acuáticas, las cuales, en la actualidad, ocupan más del $70 \%$ de ésta, siendo el buchón de agua y la elodea las especies predominantes. En cuanto a los contaminantes principales, las aguas residuales vertidas sobre el río Ubaté y las zonas altas de la cuenca que caen por escorrentía a la laguna permiten la presencia de ácido sulfhídrico, generando problemas ambientales importantes. Las consecuencias del aumento de las plantas acuáticas, se reflejan en una reducción en la capacidad de almacenamiento de la laguna, un deterioro de la calidad del agua por eutrofización y anoxia, un bloqueo de la entrada (caños) y salida de agua (río Suárez) (JICA, 1999).

La elodea pertenece a la familia Hidrocaritaceae y se desarrolla con facilidad en aguas dulces y niveles adecuados de nutrientes (Mondragón E Parada, 1996). Por su competitividad desplaza a las especies nativas y prolifera de manera no deseada. Su contenido de nutrientes es alto y la planta se puede emplear para la producción de abonos y de forrajes (CAR, 2000; Mondragón E Parada, 1996; Nacif et al. 2007). Un exceso de hierro puede disminuir su población (Pinochet et al. 2004) y, posiblemente, la alta radiación solar logra eliminar la planta (Ramírez et al. 2006). Una contaminación por exceso de elementos menores la puede igualmente afectar (Lora, 2007).

Los abonos orgánicos suministran algunos elementos esenciales y mejoran algunas propiedades físicas, químicas y biológicas del suelo (Correa \& García, 2000; Gómez, 2000; Rosas, 2002, Zapata, 2005). En el proceso de compostaje, se libera energía en forma de calor (Smith, 1997) y la elevación de la temperatura ocurre durante varias fases (Peixoto, 1988): mesofílica, termofílica, de enfriamiento y de maduración. De acuerdo a varios autores, el contenido óptimo de humedad durante el compostaje es de 40 a 60\% (Cegarra, 1994; Wilson et al. 1983; Correa E García, 2000).

El suministro de aire en la pila de compostaje provisiona oxígeno a los microorganismos y elimina el anhídrido carbónico (Jacoksen, 1994). Mediante el volteado continuo de la pila de materiales durante el proceso, se produce una aireación más homogénea (González, 1995). En la etapa inicial, el $\mathrm{pH}$ es de 5 a 6 pero, posteriormente, se incrementa hasta valores de 7 a 8 y se estabiliza alrededor de 7,0 al final (Cegarra, 1994). 
El mismo autor, indica que en relación a las materias orgánicas empleadas en la producción de compost es recomendable que contengan una relación $\mathrm{C}: \mathrm{N}$ de 25:35.

La Lombriz Roja Californiana (Eisenia foetida) (LRC) es la más conocida y empleada en más del $80 \%$ de los criaderos del mundo. La humedad adecuada para su desarrollo es del 60-70\%, el rango de temperatura es de $12-30^{\circ} \mathrm{C}$, el pH óptimo es de cinco a ocho, la aireación es fundamental para la correcta respiración y desarrollo de las lombrices; el alimento principal es materia orgánica parcial o totalmente descompuesta, constituida por residuos vegetales, estiércoles, frutas, tubérculos y restos de aserraderos. El lombricompuesto es un fertilizante orgánico de buena calidad, cuya característica fundamental es la bioestabilidad, pues no da a lugar a fermentación o putrefacción. Se debe tener en cuenta que las temperaturas elevadas, los niveles de $\mathrm{pH}$ extremos, al igual que los gases tóxicos que emanan del estiércol durante los procesos de fermentación son letales para las lombrices, por lo cual, el material orgánico, empleado como alimento, debe estar total o parcialmente descompuesto (García E Solano, 2005).

Para saber la calidad y caracterizar un producto orgánico para uso agrícola como fertilizante, el INCONTEC (Instituto Colombiano de Normas Técnicas) en compañía del ICA (Instituto Colombiano Agropecuario) diseñaron métodos de ensayo para la determinación cuantitativa de macronutrientes, de micronutrientes y de otros en abonos orgánicos o fertilizantes. La norma NTC 5167 (ICONTEC, 1997b) tiene por objeto establecer los requisitos que deben cumplir y los ensayos a los cuales deben ser sometidos los productos orgánicos usados como abonos o fertilizantes y como enmiendas del suelo. De acuerdo con esta norma, los parámetros a caracterizar son: humedad máxima $20 \%$, para materiales de origen animal y de máximo $35 \%$, para material vegetal; carbono orgánico oxidable mínimo $15 \%$ y, $\mathrm{N}, \mathrm{P}_{2} \mathrm{O}_{5}$ y $\mathrm{K}_{2} \mathrm{O}$ totales cada uno mayor de $1 \%$. Los parámetros a garantizar en base húmeda son: humedad máxima (\%), $\mathrm{N}$ total (\%) y otros como $\mathrm{P}_{2} \mathrm{O}_{5}$, carbono orgánico oxidable y $\mathrm{K}_{2} \mathrm{O}$.

El objetivo de la presente investigación fue producir seis abonos orgánicos a partir de la elodea presente en la laguna de Fúquene y evaluar estos materiales en un bioensayo, bajo condiciones de invernadero.

\section{MATERIALES Y MÉTODOS}

En la población de Susa (Cundinamarca), a 2560msnm, se produjo el compost y el lombricompuesto y, además, se realizó el bioensayo, bajo condiciones de invernadero, para evaluar los abonos orgánicos producidos. Las materias primas empleadas fueron: elodea ( $E$. densa), de la laguna de Fúquene; cal dolómitica, con 55\% de $\mathrm{CaCO}_{3}$ y $33 \%$ de $\mathrm{MgCO}_{3}$; gallinaza de jaula de gallina ponedora; levadura de uso en panadería, que ayuda a degradar proteínas complejas y carbohidratos y producen sustancias bioactivas como vitaminas, hormonas y enzimas $y$, finalmente melaza o melote, procedente de la fabricación de panela, cuya función es activar el proceso de descomposición. La elodea recolectada, se secó a temperatura ambiente durante 24 horas y, posteriormente, fue reducida a un tamaño de $2 \mathrm{~cm}$. Para el montaje de las pilas de compostaje, se emplearon cajones de madera de $2 \mathrm{~m}^{3}$ de capacidad, colocados en el piso del invernadero, aproximadamente, a $30 \mathrm{~cm}$ de altura; como se obtuvieron tres abonos orgánicos por compostaje tipo Bocashi y tres abonos orgánicos tipo lombricompuesto, se procedió así:

Formulaciones 1,2,3 por compostación: En los cajones de madera construidos, se montaron las formulaciones por duplicado agregando capas sucesivas de cada materia prima. Formulación 1. Capas: 1) Elodea 25kg; 2) Cal dolomítica $10 \mathrm{~kg}$; 3) Levadura 2,5kg; 4) Gallinaza 10kg; 5.) Melaza 2,5kg; 6) Elodea 25kg; 7) Cal dolomítica 10kg; 8) Levadura 2,5kg; 9) Gallinaza $10 \mathrm{~kg}$ y 10) Melaza 2,5kg. Formulación 2. Capas: 1) Elodea $30 \mathrm{~kg}$; 2) Cal dolomítica 5kg; 3) Levadura 1,5kg; 4) Gallinaza 9,5kg; 5) Melaza $4 \mathrm{~kg}$; 6) Elodea $30 \mathrm{~kg}$; 7) Cal dolomítica 5kg; 8) Levadura 1,5kg; 9) Gallinaza 9,5kg y 10) Melaza 4kg. Formulación 3. Capas: 1. Elodea 35kg; 2) Cal dolomítica 7,5kg; 3) Levadura 1kg; 4) Gallinaza 5kg; 5) Melaza 1,5kg; 6) Elodea 35kg; 7) Cal dolomítica 7,5kg; 8) Levadura $1 \mathrm{~kg}$; 9) Gallinaza 5kg y 10) Melaza $1,5 \mathrm{~kg}$.

Al final, se homogenizó la mezcla de cada cajón y se determinó el pH y la humedad inicial. Cada cinco días, se hicieron volteos para un total de cinco a ocho vueltas por pila; la temperatura y el $\mathrm{pH}$, se midieron cada cinco días y la humedad, se mantuvo entre 40-60\%, en cada formulación. Una vez maduros los compost (día 65), se extendieron y voltearon hasta obtener una humedad menor de 35\%. Se molieron, se tamizaron, se empacaron y se sellaron en bultos. Se tomaron 
muestras por duplicado de cada compost para realizar su análisis químico y el restante, se guardó para realizar el bioensayo. De acuerdo con las Normas Técnicas Colombianas (ICONTEC, 1997a) y los procedimientos implementados en el ICA, se realizó para cada compost el análisis de: N total (NTC-370); P total (NTC-234); K total (NTC-202); Ca y Mg (NTC-1369) (ICONTEC, 1996, 1997a; b; 1998, 2001); carbono orgánico oxidable total (NTC-5167); coliformes totales (LANIA-agrobiológicos). Para la producción de los tres lombricompuestos (formulaciones 4, 5 y 6), se adecuaron las camas, con una profundidad de $40 \mathrm{~cm}$ y $1 \mathrm{~m}^{2}$ para proporcionar las condiciones adecuadas de espacio y de aire, para así garantizar el buen desempeño de la lombriz. El piso del cajón, se cubrió con lona verde para evitar el escape de las lombrices. Para la preparación de las formulación 1, 2 y 3 , se llevó a cabo el procedimiento obteniéndose los sustratos para los tres lombricompuestos. La producción de cada formulación, se ejecutó por duplicado. Durante 40 días, se monitoreó el $\mathrm{pH}$ y la temperatura de cada muestra. La humedad, se ajustó adicionando una mezcla al $10 \%$ de agua y melaza. Obtenidas las condiciones requeridas de humedad y de temperatura, se agregaron los sustratos a su respectiva cama, esparciéndolos homogéneamente y, sobre éstos, se agregaron $4 \mathrm{~kg}$ de LRC. Posteriormente, se cubrió con plástico negro, para evitar el contacto directo de la luz, debido a la fotosensibilidad de la lombriz (García E Solano, 2005) y para protegerlas de depredadores. La humedad, se mantuvo aproximadamente en $60 \%$, con el fin de garantizar el buen desempeño de la lombriz. Una vez la estructura del sustrato se tornó granular y de coloración negruzca, a los cinco días siguientes, se suspendió la adición de agua para inducir un estado de estrés en la lombriz, se amontonó el lombricompuesto y, en el espacio vacío, se adicionó nuevo alimento; en los tres días siguientes, las lombrices migraron hacia el nuevo sustrato, dejando libre el producto. El producto recogido, se extendió sobre una lona verde, para retirar el exceso de humedad; se pasó por una malla y se empacó en sacos plásticos, tomando muestras por duplicado para su análisis y el resto para el bioensayo. El contenido de $\mathrm{N}$ total, P total, K total, Ca y Mg totales y carbono orgánico oxidable total, se hizo de acuerdo con la metodología empleada para los abonos 1-2-3.

Bioensayo. En el invernadero, se evaluó la respuesta de la lechuga Simpson a la aplicación de los abonos orgánicos. El diseño estadístico empleado fue el de bloques al azar con dos repeticiones en bolsa negra plástica para $2 \mathrm{~kg}$ de suelo derivado de cenizas volcánicas de baja fertilidad, con $75 \%$ de saturación de aluminio. Los tratamientos fueron 0, 2, 4, 6 y 8t/ha de cada abono. En cada bolsa, se sembraron cuatro semillas de lechuga y el suelo se mantuvo a capacidad de campo. A los diez días de la germinación, se dejó una planta por repetición. Posiblemente, debido a las características químicas del suelo, como $75 \%$ de saturación de aluminio intercambiable y bajos contenidos de nutrimentos, no hubo germinación en el testigo a fertilización (Lora et al. 2006). A los 70 días de la germinación, se hizo la cosecha por unidad experimental y se determinó el rendimiento en base húmeda. En el laboratorio, se secó el material a $75^{\circ} \mathrm{C}$, se molió y se determinó el contenido de $\mathrm{N}, \mathrm{P}$ y K total.

\section{RESULTADOS Y DISCUSIÓN}

Para la elodea y la gallinaza empleadas, la Tabla 1 muestra la humedad, densidad, nitrógeno, fósforo, potasio y carbono orgánico. La composición de los materiales en base seca es casi similar, sobresaliendo los contenidos, relativamente elevados, de nitrógeno, de fósforo y de carbono orgánico oxidable total. Los dos materiales son básicamente las fuentes de nitrógeno, de fósforo y de potasio. Por su parte, el calcio y el magnesio son aportados, principalmente, por la cal dolomítica y, además, por la elodea y la gallinaza.

Tabla 1. Composición de la elodea y gallinaza utilizadas, base seca.

\begin{tabular}{|c|c|c|c|c|c|c|}
\hline Material & $\mathbf{N}$-Total & $\mathbf{P}_{\mathbf{2}} \mathbf{O}_{\mathbf{5}}$ Total & $\mathbf{K}_{\mathbf{2}} \mathbf{O}$ Total & $\begin{array}{c}\text { Carbono } \\
\text { orgánico total }\end{array}$ & Humedad & Densidad \\
\hline Elodea & $3,90 \%$ & $2,28 \%$ & $1,50 \%$ & $28,90 \%$ & $90 \%$ & 0,13 \\
\hline Gallinaza & $4,60 \%$ & $2,10 \%$ & $1,50 \%$ & $34,10 \%$ & $3,50 \%$ & 0,33 \\
\hline
\end{tabular}


En la Tabla 2, se observa el efecto de las seis formulaciones de abonos en su contenido de N, P, K, Ca, Mg, carbono orgánico oxidable total y de la relación $\mathrm{C}: \mathrm{N}$. El contenido de $\mathrm{N}, \mathrm{P}_{2} \mathrm{O}_{5}, \mathrm{~K}_{2} \mathrm{O}$ para los seis abonos orgánicos cumple los requisitos específicos para Abonos Orgánicos, según la NTC-5167 en Colombia. Para carbono orgánico oxidable total, con excepción de las formulaciones tres y seis, se cumple con el requisito de un contenido mínimo a $15 \%$. Básicamente, el carbono es aportado por la elodea y la gallinaza y, en menor cantidad por la melaza. El carbono es aprovechado por los microorganismos, cuya actividad, bajo condiciones aeróbicas, esta articulada al proceso de compostación y de lombricompostación (Smith, 1997; Rosas, 2002; Muñoz, 1994; García \& Solano, 2005). Los datos muestran la importancia de las fuentes utilizadas y de la proporción de estas en la formulación (Melgarejo, 1997; Burbano, 1994; Barrera, 2003). El contenido de
$\mathrm{N}, \mathrm{P}_{2} \mathrm{O}_{5}, \mathrm{~K}_{2} \mathrm{O}, \mathrm{CaO}, \mathrm{MgO}$ y carbono presentó efecto significativo entre los abonos, debido, probablemente, al contenido y relación de los componentes empleados. Referente a la relación C: $\mathrm{N}$, se presentaron valores entre 8,9 y 11,80 , lo que significa una buena mineralización por parte de los microorganismos y actividad por parte de la lombriz roja californiana (Romero, 1999; Arango \& González, 1999). Respecto a organismos contaminantes, el análisis indicó que en los seis abonos no aparecieron unidades formadoras de colonias de coliformes totales, lo cual, es importante desde el punto de vista de salud del consumidor de los cultivos que utilicen los abonos orgánicos producidos. Se puede anotar que por su composición, los abonos orgánicos producidos se pueden considerar adecuadas, ya que muestran contenidos de nutrientes más elevados que muchos de los reportados en la literatura (Piraneque et al. 2005).

Tabla 2. Efecto de las formulaciones de abono orgánico en su contenido de N-P-K-Ca-Mg-C oxidable - Coliformes y relación C:N *.

\begin{tabular}{|c|c|c|c|c|c|c|c|c|}
\hline $\begin{array}{c}\text { Abonos } \\
\text { Orgánicos } \\
\text { Formulación }\end{array}$ & $\mathbf{N}(\%)$ & $\mathbf{P}_{2} \mathbf{O}_{5(\%)}$ & $\mathrm{K}_{2} \mathbf{O}(\%)$ & $\mathbf{M g O}(\%)$ & $\mathbf{C a O}(\%)$ & $\begin{array}{c}\text { Carbono } \\
\text { Orgánico } \\
(\%)\end{array}$ & $\begin{array}{c}\text { Coliformes } \\
(\text { UFC) }\end{array}$ & $\mathbf{C : N}$ \\
\hline 1 & $1,70 \mathrm{a}$ & $2,54 \mathrm{a}$ & $2,88 \mathrm{a}$ & $3,25 \mathrm{a}$ & $7,00 \mathrm{a}$ & $15,16 \mathrm{a}$ & 0 & 8,90 \\
\hline 2 & $1,60 \mathrm{~b}$ & $2,96 \mathrm{~b}$ & $3,40 \mathrm{~b}$ & $3,68 \mathrm{~b}$ & $8,43 \mathrm{~b}$ & $18,34 \mathrm{~b}$ & 0 & 11,50 \\
\hline 3 & $1,50 \mathrm{c}$ & $2,66 \mathrm{c}$ & $3,06 \mathrm{c}$ & $2,50 \mathrm{~d}$ & $5,71 \mathrm{c}$ & $13,30 \mathrm{c}$ & 0 & 8,90 \\
\hline 4 & $1,40 \mathrm{~d}$ & $2,29 \mathrm{~d}$ & $2,26 \mathrm{~d}$ & $2,63 \mathrm{~d}$ & $4,11 \mathrm{~d}$ & $15,14 \mathrm{a}$ & 0 & 10,80 \\
\hline 5 & $1,51 \mathrm{c}$ & $2,01 \mathrm{e}$ & $2,31 \mathrm{e}$ & $2,15 \mathrm{c}$ & $5,43 \mathrm{e}$ & $15,64 \mathrm{a}$ & 0 & 10,40 \\
\hline 6 & $1,33 \mathrm{f}$ & $2,00 \mathrm{e}$ & $2,30 \mathrm{e}$ & $2,36 \mathrm{~d}$ & $5,66 \mathrm{c}$ & $12,05 \mathrm{~d}$ & 0 & 9,10 \\
\hline
\end{tabular}

* Números con las mismas letras no presentan diferencias estadísticas significativas (Duncan $p<0,05$ ). $R^{2}: 0,85$

En la Tabla 3 aparece el efecto de dosis de las formulaciones en el rendimiento de la lechuga en base húmeda. Para las seis formulaciones hubo diferencia significativa, siendo la formulación 3 la que presentó el más alto rendimiento, debido, posiblemente, a un mayor contenido y adecuado balance de los nutrimentos presentes en el fertilizante. Por su parte, para las formulaciones producidas por acción de la LRC, la cuatro fue la de mayor producción de lechuga, debido, posiblemente, al mayor contenido disponible de nutrientes. En relación con las dosis de abono empleadas hubo efecto significativo entre las mismas, siendo la de $8 \mathrm{t}$ ha- ${ }^{1}$ la que produjo el máximo rendimiento, lo cual, muestra que la aplicación de dosis altas de abonos orgánicos se refleja en los mayores rendimientos (Lora E Caicedo, 2006; Lora et al. 2006), debido al mayor aporte de nutrimentos y, posiblemente, de biomejoradores, es decir, organismos en especial microorganismos que pueden mejorar algunas propiedades físicas y químicas del suelo, en especial en andisoles, como el empleado 
en esta investigación. En relación al contenido de N, P, $K$ en la planta indicadora, en la tabla 4 aparece el efecto de fuentes y dosis en este parámetro. En general, hubo efecto significativo de dosis y de fuentes en el contenido de estos tres nutrimentos en la planta indicadora, presentándose las mayores concentraciones con las dosis más altas bajo estudio, lo cual, está de acuerdo con lo reportado por varios investigadores (Malavolta, 1994; Osorio, 2003; Marshner, 2003). Igualmente, hubo realción entre algunos abonos orgánicos con el contenido de estos nutrientes en la planta, que guarda relación con el contenido en el abono orgánico correspondiente. En la tabla 5 es interesante anotar, que para la fuente o abono orgánico tres que fue donde se produjo el mayor rendimiento, el contenido de $\mathrm{N}, \mathrm{P}, \mathrm{K}$ en el material foliar fue de $2,010 \%, 0,153 \%$ y $1,789 \%$ para el nivel de $8 \mathrm{t}$ ha- ${ }^{1}$, donde se obtuvo el mayor rendimiento, que es estadísticamente diferente con los otros tres niveles bajo estudio, al igual que los contenidos de $\mathrm{N}$, $\mathrm{P}, \mathrm{K}$ en la lechuga. Por su parte, la relación entre los tres nutrimentos fue de 13,14:1:11,70 indicando que, en general, debe haber una relación entre los nutrimentos foliares y el rendimiento (Malavota, 1994; Sadeghian et al. 2006; Kirkby \& Römheld, 2007; Epstein E Bloom, 2004; Mengel E Kirkby, 2001). En general, la relación $\mathrm{N}: \mathrm{K}$ fue de uno, independientemente, de la dosis de abono aplicada.
El comportamiento de la temperatura durante el proceso de compostación fue considerado normal; la compostación se inicio a los $20^{\circ} \mathrm{C}$, aumentó a $65^{\circ} \mathrm{C}$ a los diez días, para luego declinar y establecerse nuevamente en $20^{\circ} \mathrm{C}$ a los 55 días. Las lombrices, se adicionaron a los 45 días con una temperatura de $30^{\circ} \mathrm{C}$. Así, las fases mesofílica, termofílica, de enfriamiento y maduración se comportaron de manera similar a lo expuesto por Peixoto (1988); Correa E García (2000) y Farías (1997). Las tres formulaciones, bajo estudio, cumplen con las fases anteriores, indicando que los componentes y las proporciones de los mismos son adecuados. Treinta y cinco días después de la incorporación de la lombriz, se encontró que los tres lombricompuestos (abonos 4, 5 y 6) estaban listos para ser utilizados. El pH durante el proceso, se mantuvo entre 6 y 8 , que es el óptimo para que la lombriz cumpla con su función de transformación. Para las formulaciones 1,2 y 3 , el pH mostró una evolución, acorde con un buen proceso de compostaje (Cegarra, 1994). En la etapa inicial, el pH desciende, pero luego se incrementa por la liberación de amonio durante la etapa termofílica del proceso y, posteriormente, vuelve a bajar hasta valores cercanos a la neutralidad. El pH final de los abonos obtenidos estuvo alrededor de 7, lo cual, está de acuerdo con la normatividad Colombiana (NTC-5167).

Tabla 3. Efecto de formulaciones y dosis en el rendimiento $(\mathrm{g})$ de la planta indicadora, base húmeda *

\begin{tabular}{|c|c|c|c|c|c|}
\hline \multirow{2}{*}{ Formulación } & \multicolumn{5}{|c|}{ Dosis t ha-1 $^{-1}$} \\
\cline { 2 - 6 } & 2 & 4 & 6 & 8 & $Y$ \\
\hline 1 & $0,892 \mathrm{a}$ & $3,804 \mathrm{a}$ & $6,957 \mathrm{a}$ & $9,998 \mathrm{a}$ & $5,410 \mathrm{~A}$ \\
\hline 2 & $2,685 \mathrm{~b}$ & $3,800 \mathrm{a}$ & $6,888 \mathrm{a}$ & $16,958 \mathrm{~b}$ & $7,583 \mathrm{~B}$ \\
\hline 3 & $1,408 \mathrm{c}$ & $1,796 \mathrm{c}$ & $6,893 \mathrm{a}$ & $23,429 \mathrm{c}$ & $8,382 \mathrm{C}$ \\
\hline 4 & $2,416 \mathrm{~b}$ & $3,792 \mathrm{a}$ & $4,220 \mathrm{~b}$ & $21,305 \mathrm{~d}$ & $7,933 \mathrm{~B}$ \\
\hline 5 & $0,344 \mathrm{~d}$ & $1,799 \mathrm{c}$ & $5,198 \mathrm{c}$ & $18,369 \mathrm{~b}$ & $6,427 \mathrm{D}$ \\
\hline 6 & $1,902 \mathrm{e}$ & $3,505 \mathrm{~b}$ & $4,366 \mathrm{~b}$ & $6,898 \mathrm{e}$ & $4,167 \mathrm{E}$ \\
\hline$Y_{j}$ & $1,608 \mathrm{~A}$ & $3,083 \mathrm{~B}$ & $5,754 \mathrm{C}$ & $16,158 \mathrm{D}$ & 6,55 \\
\hline
\end{tabular}

* Números con las mismas letras no difieren estadísticamente (Duncan $p<0,05)$. R²: 0,80. Y,Yj: Promedios 
Tabla 4. Efecto de formulaciones y dosis en el contenido de nitrógeno, fósforo y potasio en la planta indicadora en base seca *.

\begin{tabular}{|c|c|c|c|c|}
\hline Formulación & Dosis (t ha-1) & $P(\%)$ & $\mathrm{N}(\%)$ & $K(\%)$ \\
\hline \multirow{4}{*}{1} & 2,0 & $0,016 a$ & $0,046 \mathrm{a}$ & $0,109 a$ \\
\hline & 4,0 & $0,027 \mathrm{~b}$ & $0,190 \mathrm{~b}$ & $0,421 b$ \\
\hline & 6,0 & $0,051 \mathrm{c}$ & $0,356 \mathrm{c}$ & $0,776 \mathrm{c}$ \\
\hline & 8,0 & $0,075 d$ & $0,549 d$ & $1,135 d$ \\
\hline \multirow{4}{*}{2} & 2,0 & $0,017 \mathrm{a}$ & $0,201 \mathrm{a}$ & $0,331 \mathrm{a}$ \\
\hline & 4,0 & $0,024 \mathrm{~b}$ & $0,278 b$ & $0,432 b$ \\
\hline & 6,0 & $0,046 \mathrm{c}$ & $0,523 \mathrm{c}$ & $0,779 \mathrm{c}$ \\
\hline & 8,0 & $0,099 \mathrm{e}$ & $1,303 d$ & $1,960 \mathrm{~d}$ \\
\hline \multirow{4}{*}{3} & 2,0 & $0,020 a$ & $0,117 a$ & $0,111 \mathrm{a}$ \\
\hline & 4,0 & $0,032 \mathrm{~b}$ & $0,153 \mathrm{a}$ & $0,132 a$ \\
\hline & 6,0 & $0,046 \mathrm{c}$ & $0,592 b$ & $0,522 b$ \\
\hline & 8,0 & $0,153 \mathrm{f}$ & $2,010 \mathrm{c}$ & $1,789 \mathrm{c}$ \\
\hline \multirow{4}{*}{4} & 2,0 & $0,015 a$ & $0,104 \mathrm{a}$ & $0,211 \mathrm{a}$ \\
\hline & 4,0 & $0,025 \mathrm{~b}$ & $0,162 \mathrm{~b}$ & $0,323 \mathrm{~b}$ \\
\hline & 6,0 & $0,037 \mathrm{c}$ & $0,184 b$ & $0,355 \mathrm{~b}$ \\
\hline & 8,0 & $0,138 \mathrm{f}$ & $0,930 \mathrm{c}$ & $1,790 \mathrm{c}$ \\
\hline \multirow{4}{*}{5} & 2,0 & $0,014 \mathrm{a}$ & $0,015 \mathrm{a}$ & $0,031 \mathrm{a}$ \\
\hline & 4,0 & $0,022 \mathrm{~b}$ & $0,055 b$ & $0,171 \mathrm{~b}$ \\
\hline & 6,0 & $0,038 \mathrm{c}$ & $0,156 \mathrm{c}$ & $0,511 \mathrm{c}$ \\
\hline & 8,0 & $0,139 \mathrm{f}$ & $0,551 \mathrm{~d}$ & $1,821 d$ \\
\hline \multirow{4}{*}{6} & 2,0 & $0,028 \mathrm{a}$ & $0,159 a$ & $0,351 \mathrm{a}$ \\
\hline & 4,0 & $0,055 \mathrm{~b}$ & $0,317 \mathrm{~b}$ & $0,653 b$ \\
\hline & 6,0 & $0,071 \mathrm{c}$ & $0,400 \mathrm{c}$ & $0,844 \mathrm{c}$ \\
\hline & 8,0 & $0,109 \mathrm{e}$ & $0,626 \mathrm{~d}$ & $1,291 d$ \\
\hline
\end{tabular}

${ }^{*}$ Números con las mismas letras no difieren estadísticamente (Duncan $\left.p<0,05\right) . R^{2}: 0,80$.

Tabla 5. Efecto de dosis en el rendimiento, contenido y relación N:P:K de la lechuga para la formulación 3*.

\begin{tabular}{|c|c|c|c|c|c|}
\hline \multirow{2}{*}{$\begin{array}{c}\text { Dosis } \\
\text { (t/ha-1) }\end{array}$} & \multirow{2}{*}{$\begin{array}{c}\text { Rendimiento } \\
\text { (g/planta) }\end{array}$} & \multicolumn{3}{|c|}{ Concentración (\%) } & Relación \\
\cline { 3 - 6 } & & $\mathbf{N}$ & $\mathbf{P}$ & $\mathbf{K}$ & $\mathbf{N}: \mathbf{P}: \mathbf{K}$ \\
\hline 2 & $1,408 \mathrm{a}$ & $0,117 \mathrm{a}$ & $0,020 \mathrm{a}$ & $0,111 \mathrm{a}$ & $5,85: 1: 5,55$ \\
\hline 4 & $1,796 \mathrm{~b}$ & $0,153 \mathrm{ab}$ & $0,032 \mathrm{~b}$ & $0,132 \mathrm{~b}$ & $4,78: 1: 4,13$ \\
\hline 6 & $6,893 \mathrm{c}$ & $0,592 \mathrm{c}$ & $0,046 \mathrm{c}$ & $0,522 \mathrm{c}$ & $12,87: 1: 11,35$ \\
\hline 8 & $23,429 \mathrm{~d}$ & $2,010 \mathrm{~d}$ & $0,153 \mathrm{~d}$ & $1,789 \mathrm{~d}$ & $13,14: 1: 11,70$ \\
\hline
\end{tabular}

* Números con las mismas letras no difieren estadísticamente (Duncan $p<0,05)$. R: 0,850. 


\section{CONCLUSIONES}

Bajo las condiciones de la presente investigación, se puede concluir:

Con los procesos utilizados, se pueden obtener abonos orgánicos empleando la planta acuática elodea (Egeria densa).

El rendimiento de la planta indicadora utilizada en el bioensayo dependió de la dosis y de las fuentes empleadas, siendo la fuente tres la mejor opción.

De acuerdo con los requisitos exigidos por la normatividad colombiana para abonos orgánicos, las formulaciones obtenidas cumplen con estos parámetros.

La producción de abonos orgánicos a partir de la "maleza" acuática (Egeria densa) y su empleo en la agricultura puede ser una buena opción para mantener, adecuadamente, el espejo de agua de lagunas invadidas.

\section{BIBLIOGRAFÍA}

ARANGO, G.; GONZÁLEZ, H. 1999. Cambios químicos durante la compostación de residuos de cosecha. Suelos Ecuatoriales. 29(1):25-31.

BARRERA, L. 2003. El papel de la materia orgánica en el manejo integral de la fertilidad del suelo. En: Manejo Integral de la Fertilidad del Suelo. Soc. Col. Ciencia del Suelo. Bogotá, D.C., p.123-134.

BURBANO, H. 1994. La materia orgánica del suelo en el contexto de una agricultura sostenible. En: Fertilidad del Suelo, Diagnóstico y Control. Soc. Col. de la Ciencia del Suelo. Bogotá, D.C., p.187217.

CEGARRA, J. 1994. Compost de desechos orgánicos y criterio de calidad del compost. Memoria del VII Congreso Colombiano de la Ciencia del Suelo. Soc. Col. de la Ciencia del Suelo, Bogotá, D.C. $325 p$.

CORPORACIÓN AUTÓNOMA REGIONAL CAR. 2000. Fúquene: El lecho de la zorra. Primera Ed. D’vinni. Editorial LTDA. Bogotá, D.C. 378p.

CORREA, L.; GARCÍA, L. 2000. Evaluación fisicoquímica y microbiológica de compostaje de mortalidad y gallinaza como alternativa ambiental de los residuos de la industria avícola. Trabajo de grado Químico Industrial. Convenio UDCA-CTB. Bogotá. 310p.

EPSTEIN, E.; BLOOM, A.J. 2004. Mineral Nutrition of Plants: Principles and perspectives. $2^{\text {nd }}$ ed. Sunderland: Sinauer Associates. Massachusetts, USA. p.201-241.

FARÍAS, D. 1997. Caracterización química de un proceso de compostaje. Tesis Química Universidad Nacional de Colombia. Bogotá. 120p.

GARCÍA, C.; SOLANO, F. 2005. Cría de la lombriz de tierra una alternativa ecológica y rentable. Universidad de Cundinamarca. Ubaté. 316p.

GÓMEZ, J. 2000. Abonos orgánicos, Compostaje, Sustrato, Humus líquido, Lombricompuesto. En: Laboratorio Nacional Insumos Agrícolas, ICA. Mosquera, Cundinamarca. 215p.

GONZÁLEZ, A. 1995. El compost, fertilizante orgánico del futuro. Universidad Nacional de Colombia. Bogotá, D.C. 771p.

INSTITUTO COLOMBIANO DE NORMAS TÉCNICAS ICONTEC. 1996. Determinación cuantitativa del fósforo. $1^{a}$ Actualización. Bogotá, D.C. NTC-234. $18 \mathrm{p}$.

INSTITUTO COLOMBIANO DE NORMAS TECNICAS. 1997a. Determinación de nitrógeno total. $3^{a}$ actualización. Bogotá, D.C. NTC-370. 7p.

INSTITUTO COLOMBIANO DE NORMAS TECNICAS. 1997b. Productos orgánicos usados como abonos o fertilizantes y enmiendas de suelos. $1^{\mathrm{a}}$ Actualización. Bogotá, D.C. NTC-5167. 27p.

INSTITUTO COLOMBIANO DE NORMAS TECNICAS. 1998. Determinación de Zn-Cu-Fe-Mn-Ca-Mg. $1^{\text {a }}$ Actualización. Bogotá, D.C. NTC-1369. 45p.

INSTITUTO COLOMBIANO DE NORMAS TECNICAS. 2001. Determinación de K en abonos o fertilizantes. $2^{a}$ Actualización. Bogotá, D.C. NTC-202. 11p. 
JICA AGENCIA JAPONESA PARA LA COOPERACIÓN INTERNACIONAL Y CORPORACIÓN AUTÓNOMA DE CUNDINAMARCA. 1999. Estudio sobre plan de mejoramiento ambiental regional para la cuenca de la Laguna de Fúquene. Informe principal de progreso. CAR. Bogotá D.C. 287p.

JACOKSEN, S. 1994. Aerobic Decomposition of Organic Wastes. Stoichiometric Calculation of Air Change. Resources Conservation and recycling. USA. 179p.

KIRKBY, E.; RÖMHELD, V. 2007. Micronutrients in plant physiology. Functions, uptake and mobility. Proceedings 543, The International Fertilizers Society. York, United Kingdom. p.10-13.

LORA. R.; CAICEDO, C. 2006. Respuesta de la papa criolla (Solanum phureja) a fuentes y dosis de abono orgánico y a un fertilizante mineral. Suelos Ecuatoriales 36(1):13-18.

LORA, R.; PULIDO, I.; MÉNDEZ, A.; PEÑA, F. 2006. Efecto de la fertilización orgánica y mineral en rendimiento de lechuga (Lactuca sativa L.) en un suelo Typic Hapludalfs de mediana fertilidad. Rev. U.D.C.A Act. \& Div. Científica (Colombia). 9(1):107-116.

LORA, R. 2007. Contaminación por elementos menores y posibles soluciones. Rev. U.D.C.A Act. E Div. Científica. 10(1):5-20.

MALAVOLTA, E. 1994. Diagnóstico foliar. Fertilidad de Suelos, Diagnóstico y Control. Soc. Col. de la Ciencia del Suelo. Bogotá. D.C. p.57-98.

MARSHNER, H. 2003. Mineral nutrition of higher plants. $2^{\text {nd }}$. Ed. Academic Press. San Diego, California. USA. 889p.

MELGAREJO, P. 1997. Evaluación de parámetros de calidad en humus de lombriz y compost. Trabajo de grado Químico. Universidad Nacional de Colombia. Bogotá, D.C. 110p.

MENGEL, K.; KIRKBY, E.A. 2001. Principles of plant nutrition. Kluwer Academic Publishers. 849p.
MONDRAGÓN, V.; PARADA, R. 1996. Utilización de la Elodea brasilera Egeria densa, como complemento alimenticio de la ceba de conejos en Boyacá. Facultad de Agronomía, Universidad Nacional. Bogotá. D.C. 115p.

MUÑ̃Z, R. 1994. Los abonos orgánicos y su uso en la agricultura. Soc. Col. de la Ciencia del Suelo. Fertilidad de suelos, diagnóstico y control. Bogotá, D.C. p.293-304.

NACIF, Y.; CÁRDENAS, R.; LATOURNERIE, J. 2007. Evaluación del proceso de descomposición aeróbica de Egeria densa como alimento potencial para especies acuáticas. REDVET, Rev. Electrónica de Veterinaria. 8(4):1-12.

OSORIO, W. 2003. Eficiencia y efectividad de la fertilización en la agricultura de Colombia. Manejo integral de la fertilidad del suelo. Soc. Col. de la Ciencia del Suelo. Bogotá. D.C. p.177-210

PEIXOTO, R. 1988. Compostagen: Opçäo para o manejo orgánico do Solo. Londrina, Brasil. IAPAR. Circular 57. 48p.

PINOCHET, D.; RAMÍREZ, C.; MAC DONALD, R.; RIEDEL, L. 2004. Concentraciones de elementos minerales en Egeria densa Planch. colectada en el Santuario de la naturaleza Carlos Anwandter, Valdivia, Chile. Agro Sur. 32(2):80-86.

PIRANEQUE, N.; AGUIRRE, S.; BAUTISTA, C. 2005. Caracterización y evaluación del Bocashi y otros biopreparados en la producción de cebolla de bulbo en condiciones semicontroladas. Suelos Ecuatoriales. 35(2):65-70.

RAMÍREZ, C.; CARRASCO, E.; MARIANI, S.; PALACIOS, N.; 2006. La desaparición de Luchecillo (Egeria densa) del Santuario del Rio Cruces (Valdivia, Chile): Una hipótesis plausible. Ciencia y Trabajo. 8(20):79-86.

ROMERO, M. 1999. Caracterización química y microbiológica de lombricompuesto producido por Glossoscolex sp. Suelos Ecuatoriales. 29(1):89-94. 
ROSAS, A, 2002. Agricultura Orgánica Práctica. En: Gómez, A. Ed. Alternativas para la agricultura del futuro. Bogotá. 283 p.

SADEGHIAN, S.; MEJÍA, B.; ARCILA, J. 2006. Composición elemental de frutos de café y extracción de nutrientes por la cosecha en la zona cafetera de Colombia. CENICAFE. 57(4):251261.

SMITH, E. 1997. Composts in the Landscape. Bulletin 8:12p.

VALDERRAMA, J.; MOSQUERA, A. 1979. Estudio de Fúquene. Corporación Autónoma Regional de la Sabana de Bogotá y de los Valles de Ubaté y Chiquinquirá. Bogotá. 198p.

WILSON G.; PARR, J.; SIKARA, L. 1983. Experiences with Organic Waste Composting in Developing Countries. Suelos FAO. 23p.

ZAPATA, R. 2005. Función de la materia orgánica sobre la movilidad iónica. Suelos Ecuatoriales. 35 (1):23-35.

Recibido: Octubre 9 de 2008

Aceptado: Abril 22 de 2009 